\title{
HL-LHC Accelerator Upgrade Project US CONTRIBUTION TO CERN: RF Dipole Crab Cavities
}

L. Ristori, P. Berrutti, M. Narduzzi - Fermi National Accelerator Laboratory, Batavia, USA With contribution from ANL, BNL, JLAB, LBNL, ODU, SLAC

\section{Introduction}

The HL-LHC Accelerator Upgrade Project (HL-LHC AUP), focuses on production of quadrupoles and cavities by sharing the work among a consortium of US Laboratories (FNAL, ANL, LBNL, BNL, JLAB and SLAC) and Old Dominion University, and in close connection with the CERN-led HL-LHC Collaboration.

Crab cavities are one of the key technologies enabling the High Luminosity upgrade of LHC by rotating bunches at the interaction point and recovering luminosity lost due to the nonzero crossing angle.

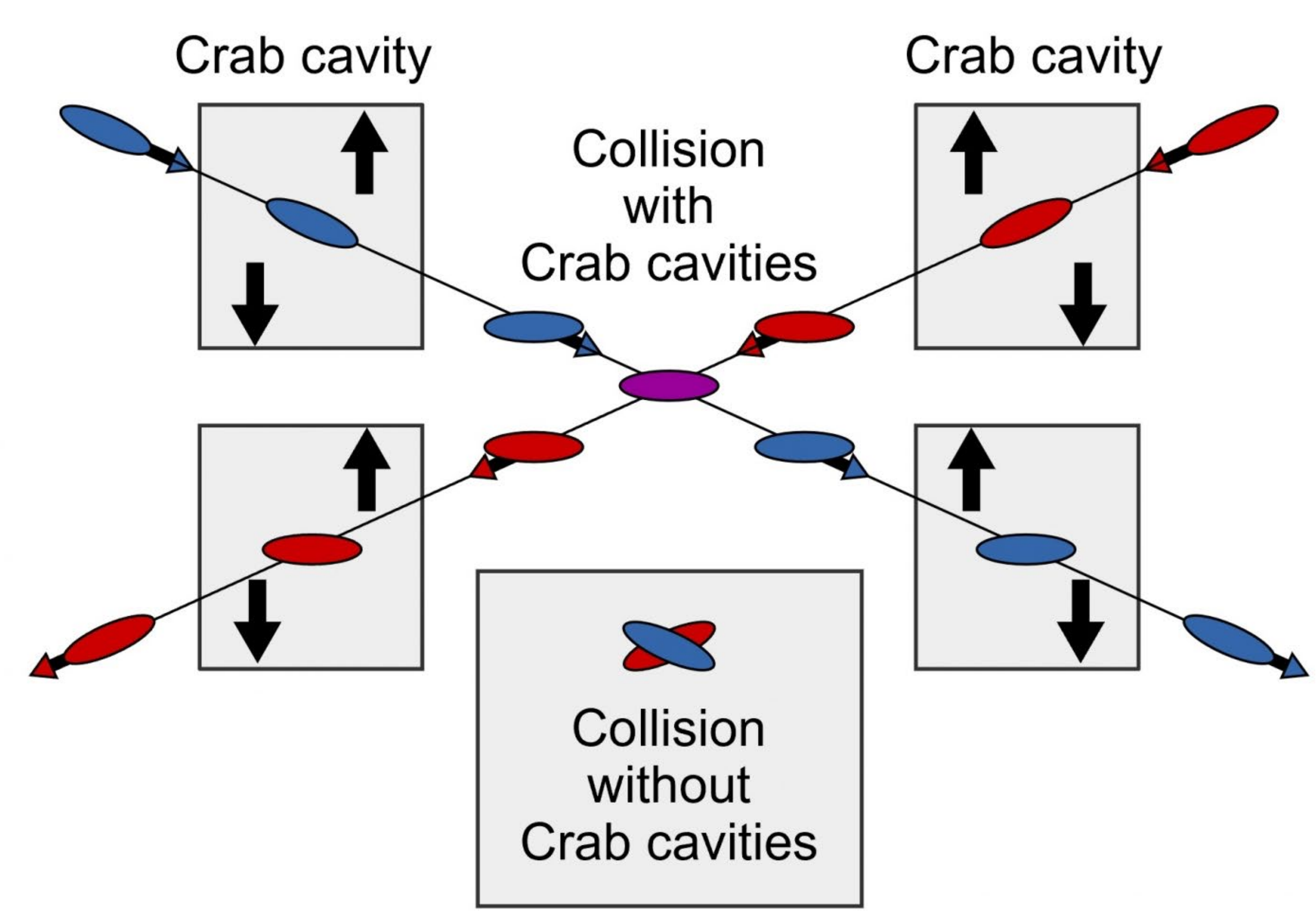

Bunch-rotation at the interaction point

\section{Design}

The design was carried out by Old Dominion University in collaboration with SLAC. The RFD design is a compact design with reduced peak surface fields and high shunt impedance. The mechanical design by CERN ensures compliance with European pressure directives.

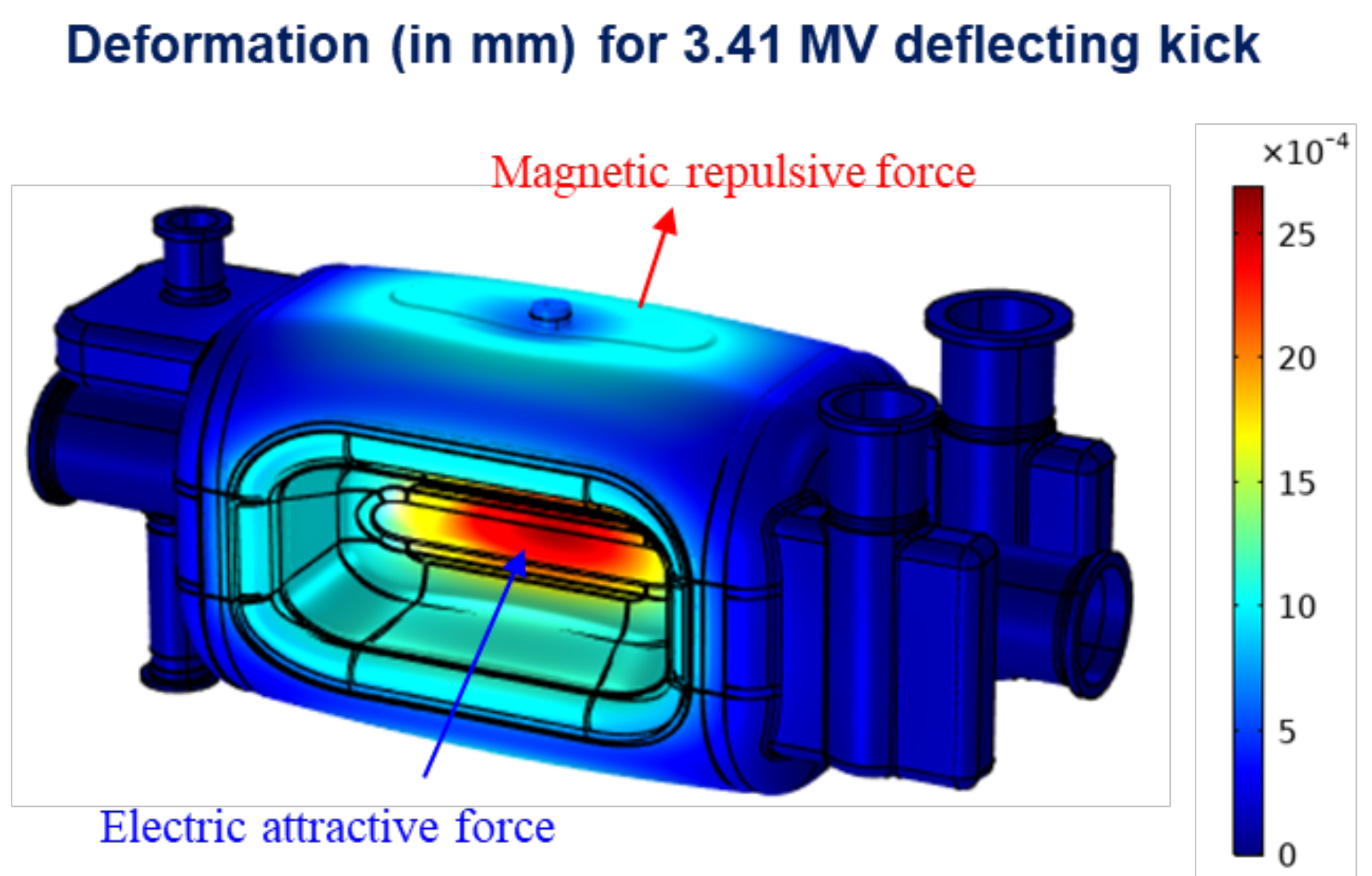

Electromagnetic design of bare cavity by AUP

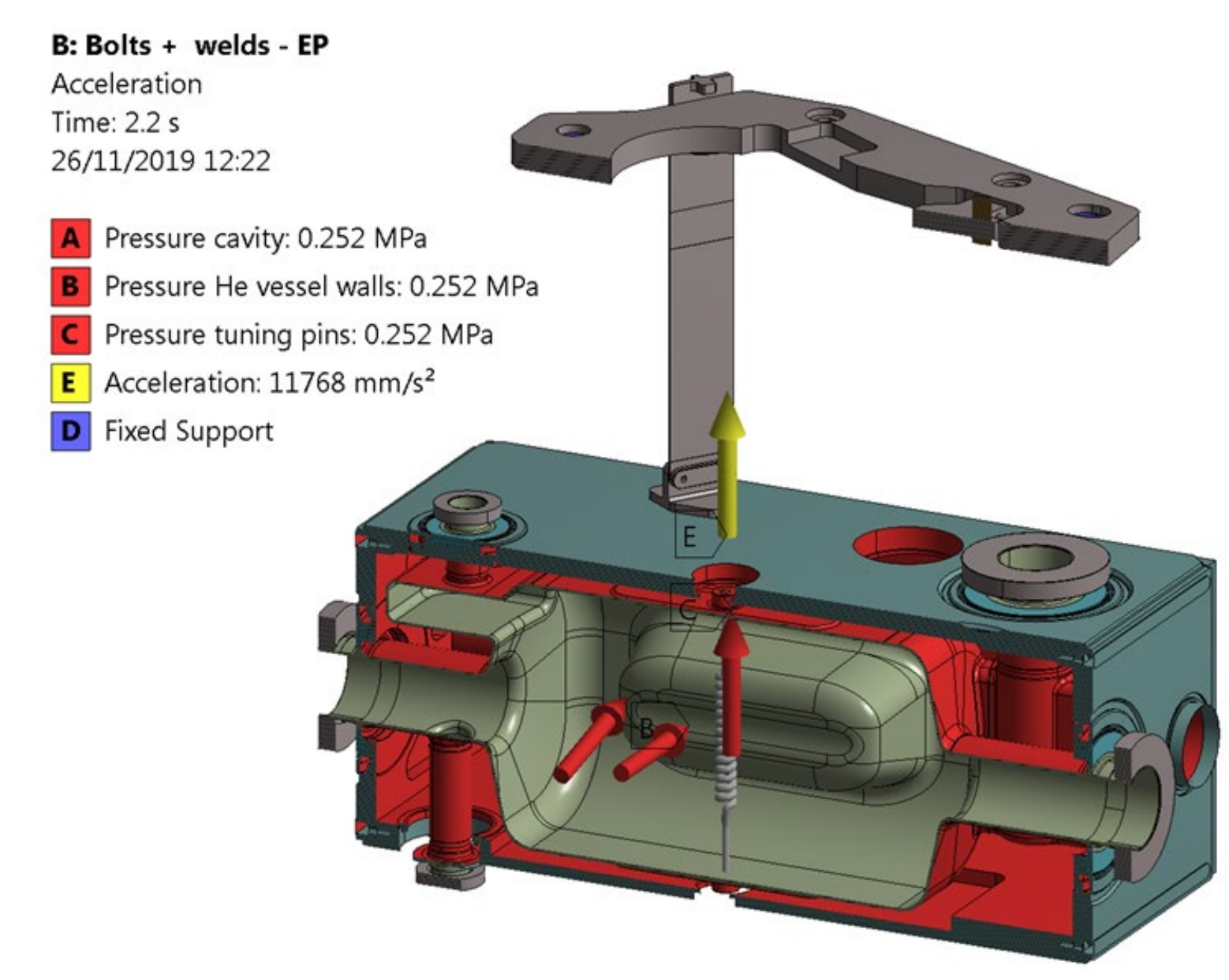

Mechanical design of dressed cavity by CERN
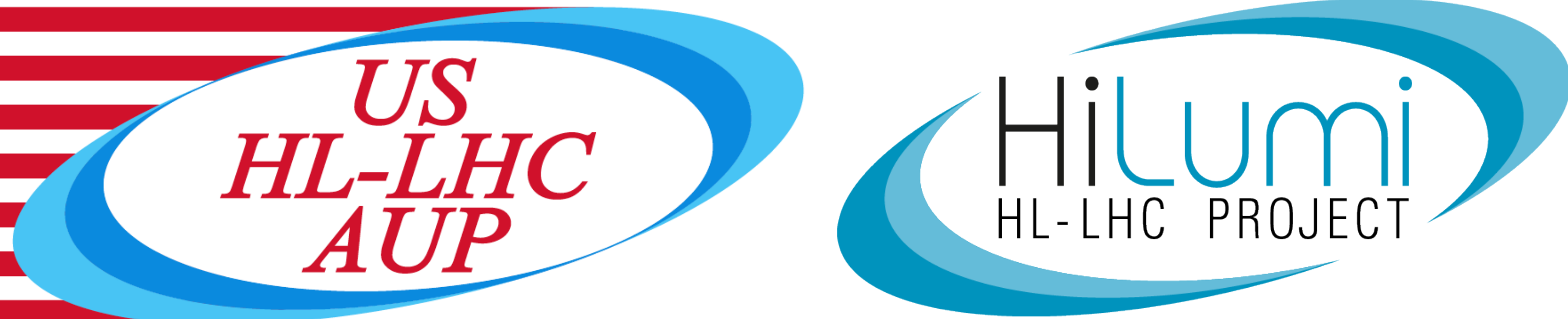

\section{Fabrication}

AUP has launched fabrication of cavities in industry with a plan to produce 2 prototypes, 2 pre-series, and 10 series cavities. Ultimately, 8 cavities will be installed in the HL-LHC tunnel. A rigorous quality assurance plan is in place to ensure compliance with stringent $C E R N$ requirements.
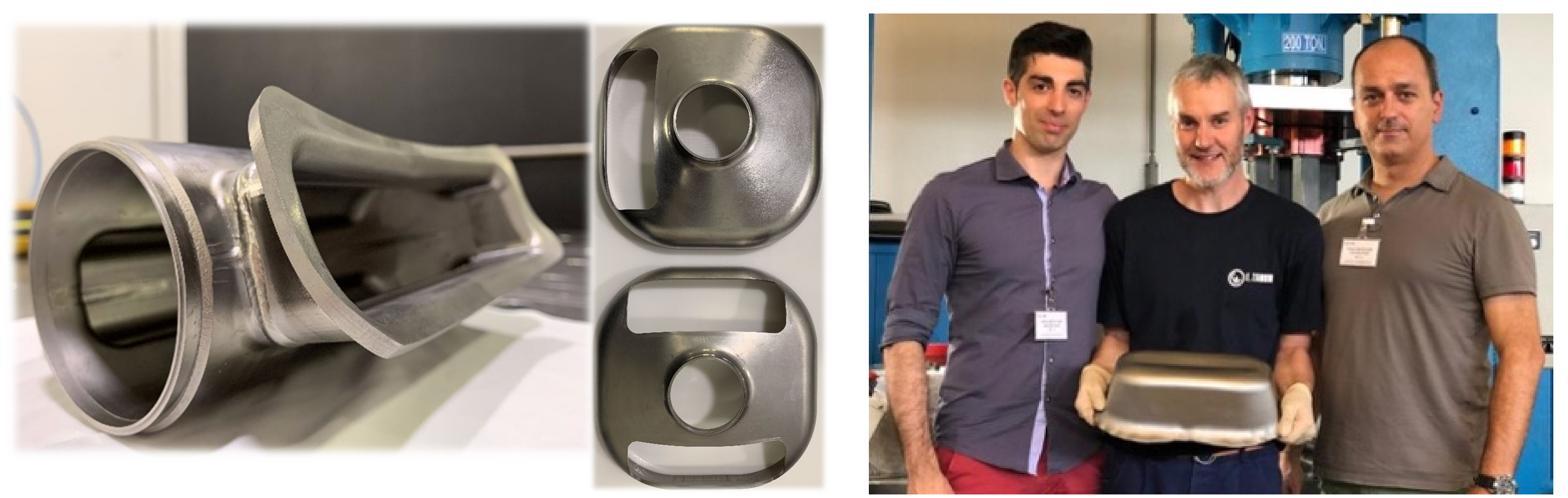

Left - Niobium components of the RFD crab cavity during forming, machining, and electron-beam welding operations. Right - successful forming of Niobium deflecting pole of cavity, the most important component in the cryomodule.

\section{Chemical Processing and Qualification}

A dedicated rotational chemical processing tool was developed at ANL and validated thanks to LARP prototypes, later inherited by AUP. Fermilab facilities were utilized for the heat treatments and cold tests of the RFD cavities.

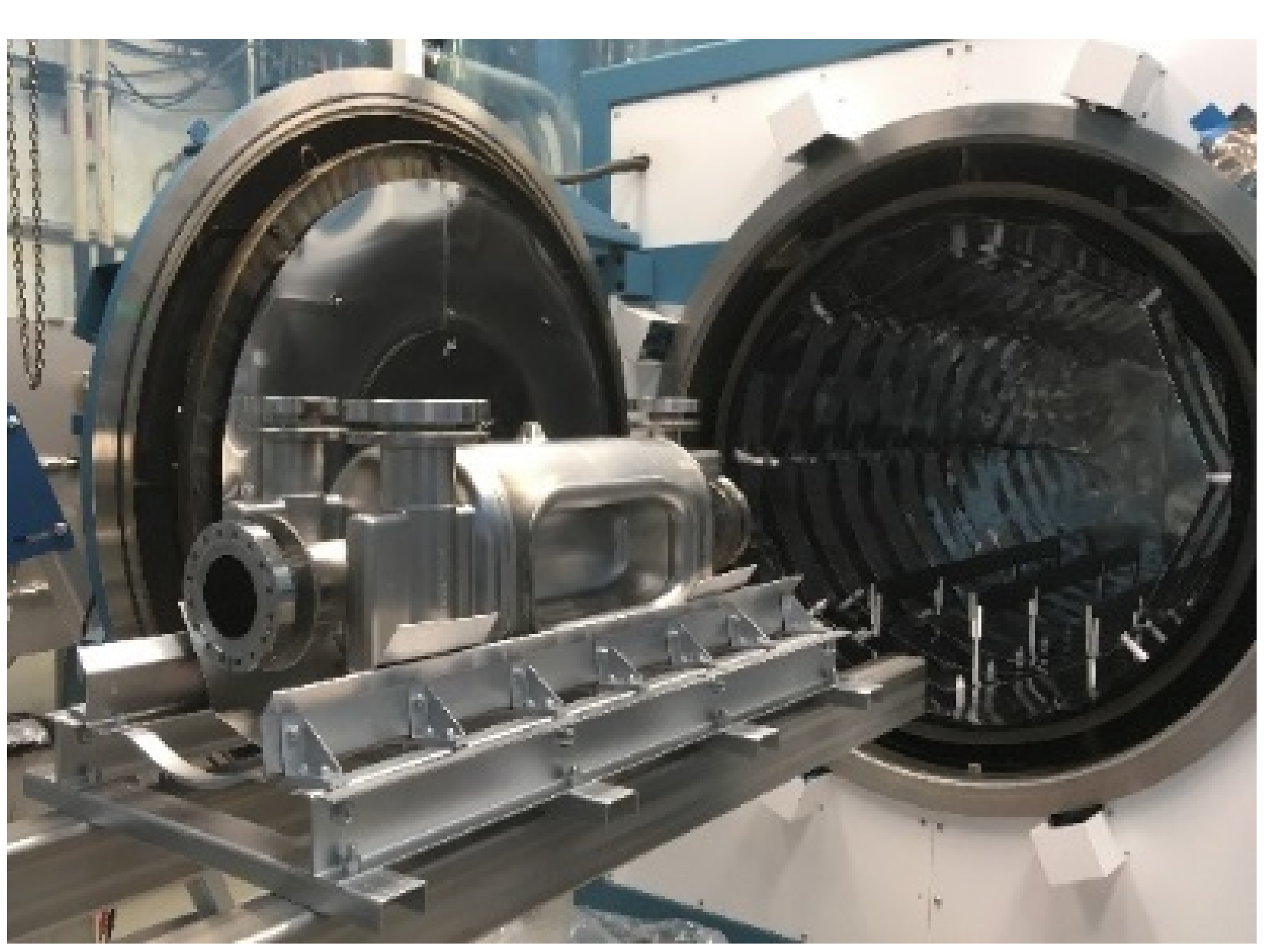

RFD bare cavity preparing to enter the high-

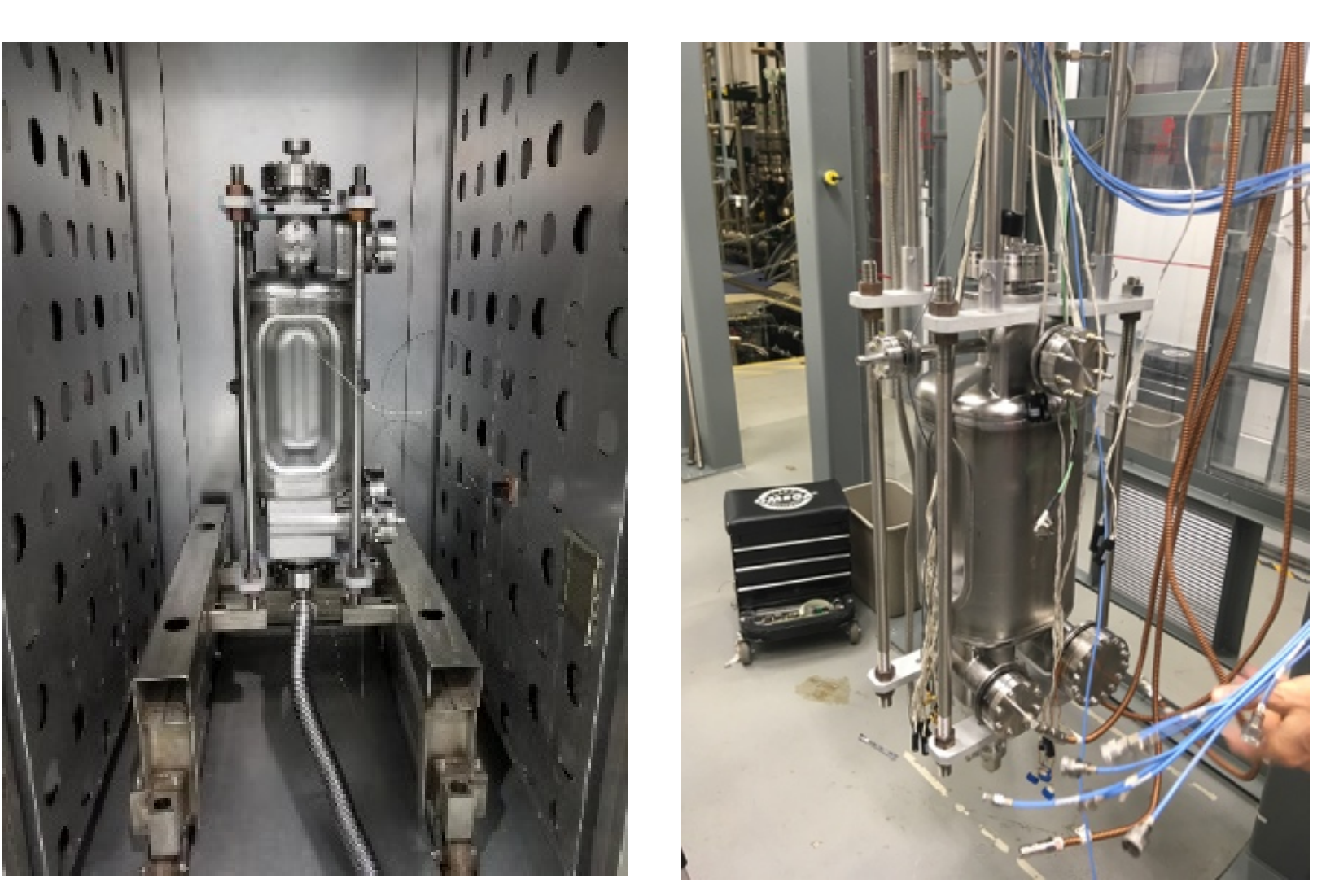

eft - Bare cavity ready for the $120^{\prime} \mathrm{C}$ lowtemperature bake. Right - Bare cavity ready for a cold test in VTS. temperature treating oven at Fermilab.

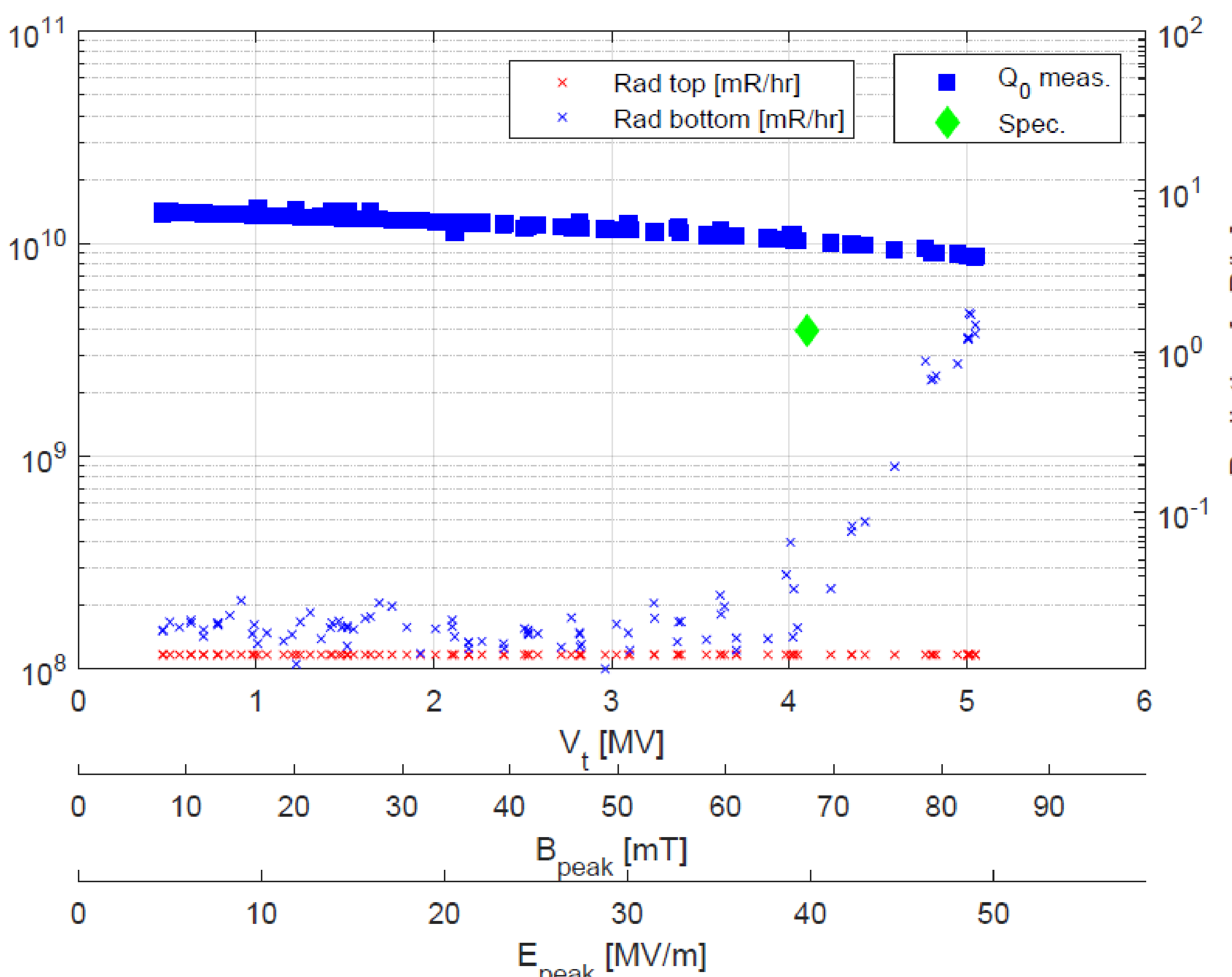

LARP prototype \#1 exceeded HL-LHC requirements at Fermilab thanks to the successful validation of dedicated facilities and procedures
This document was prepared in part using the resources of the Fermi National Accelerator Laboratory (Fermilab), a U.S. Department of energy, Office of science, HEP User Facility. Fermilab is managed by Fermi Research Alliance, LLC (FRA), acting under Contract No. DE- AC0207CH11359. 